\title{
The Influence of Individualised Supports on the Self-Efficacy of Employed Diabetics in Hawai'i: Findings from a Mixed Method Study
}

\author{
Denise L. Uehara, Christy M. Nishita, Tammy Tom and Landry Fukunaga \\ University of Hawai'i at Mānoa, Center on Disability Studies, United States
}

\begin{abstract}
Self-management is a key component of diabetes care and enhancing patient self-efficacy is an Simportant factor. Typical diabetes education programs include strategies to increase self-efficacy, but little information exists about the effectiveness of such programs within Asian and Pacific populations. The Hawai'i Demonstration to Maintain Independence and Employment was a federally funded, community-based randomised trial in which treatment group participants received individualised life coaching and pharmacist counselling over a 12-month period. The study measured changes in diabetes self-efficacy among treatment and control group participants using repeated measures analysis of covariance. Focus group findings provided a comprehensive picture of participants' perception of their experiences in the trial and more specifically the individualised intervention. There was a significant effect of the intervention on diabetes self-efficacy at the $p<.01$ level $[F(1,187)=10.40, p=$ .002]. These findings demonstrate the efficacy of individually tailored approaches to diabetes selfmanagement within a diverse, employed sample.
\end{abstract}

Keywords: self-efficacy, life coaching, pharmacist counseling, diabetes, mixed-methods

Individuals with diabetes are at an increased risk for developing related complications such as blindness, cardiovascular disease, renal failure, stroke, neuropathy and amputation. To prevent diabetes-related morbidity and mortality, persons with diabetes are expected to carry out routine disease self-management strategies (e.g., diet, exercise, self-monitoring of blood glucose, foot care and medication adherence) (Krichbaum \& Buethe, 2003; Norris, Engelgau, \& Narayan, 2001). Self-management is a key component of diabetes care and enhancing patient self-efficacy is important to improved self-management.

Managing diabetes involves long-term lifestyle changes that are affected by individual motivation and readiness to adhere to healthy behaviours. Motivation can be internal (internal desire to change) or external (external rewards or consequences of change) in nature and may vary for different ethnic/cultural groups as well as age groups (Satia \& Galanko, 2007). Particularly for adults, learning experiences to help develop new skills; challenge preconceived notions, attitudes and beliefs; help revise world views; and promote self-regulation are often utilised (Spigner-Littles \& Anderson, 1999). Self-efficacy, or the belief in one's own ability to exercise control over health behaviours, can be a strong determinant about how people motivate themselves and behave. Self-efficacy beliefs influence how people respond and behave in certain situations (Bandura, 1994). When compared with self-esteem, self-efficacy is a better predictor of comprehensive self-care and blood sugar control or haemoglobin Alc (HbAlc) levels (Johnston-Brooks, Lewis, \& Garg, 2002) and a significant association has been found between self-efficacy and self-management behaviours in an ethnically diverse population (Sarkar, Fisher, \& Schillinger, 2006).

Typical diabetes education programs include strategies to increase self-efficacy. Recommended intervention programs include multiple strategies or methods to increase self-efficacy, such as goal setting; stress management; and problem-solving of personal, physical and emotional stress. Goal setting through a collaborative

Address for correspondence: Denise L. Uehara, University of Hawai i at Mānoa Center on Disability Studies 1776 University Avenue Honolulu, HI 96822 duehara@hawaii.edu 
approach for health-related issues is one strategy used to address self-management. Goal setting related to diabetes education has its roots in social cognitive theory (Sprague, Shultz, \& Branen, 2006). It includes the process of identifying behaviours that the participant connects to meaningful outcomes, increased confidence to perform behaviours, and providing positive reinforcement believed to maximize goal attainment.

Programs designed to enhance self-efficacy among people with diabetes have demonstrated relative success across multiple populations (Krichbaum \& Buethe, 2003; Whittemore, Melkus, \& Grey, 2002). However, little information exists about such programs within Asian and Pacific Island populations. The findings from this study add to the literature on designing individualised diabetes self-management programs across a range of ethnicities.

The Hawai'i Demonstration to Maintain Independence and Employment (HI-DMIE) was a federally funded, community-based randomised trial that examined supports intended to delay or prevent diabetes-related disability, unemployment and reliance on federal assistance programs. The study was specifically funded to identify supports that would enable employed persons with diabetes to remain healthy and working. This article builds on the findings of Sarkar et al. (2006) by testing an intervention designed to support an individual's diabetes management through personalised one-to-one interactions with life coaches and pharmacists. The purpose of this article is to examine the influence of life coaching and pharmacist counselling on self-efficacy for employed diabetics. Specifically we look at the psychological impact of personalised, sustained and incentive-driven disability prevention activities. The following research questions guided this study: (1) Did the intervention improve diabetes selfefficacy? (2) Did the perceptions of the participants reflect and substantiate improvement in diabetes self-efficacy?

\section{Program Design}

The HI-DMIE intervention program consisted of one year of individualised, participant-driven support from a life coach and pharmacist. Life coaching is a systemised, structured approach that involves collaboration between a trained life coach and client with the goal of facilitating change and improving motivation. Life coaches assisted participants with setting and achieving work, wellness and personal goals of their choice; and with finding the resources, tools and information to manage their diabetes. Nine life coaches employed by the study worked with participants to increase self-efficacy by helping individuals set and track achievement of selfidentified goals. Life-coaching services were participant-driven and conversations could address lifestyle changes, health behaviours related to their diabetes or issues related to their work or employment.
The HI-DMIE life coaching model developed by project staff used executive, life and health coaching as a foundation. The International Coaching Federation (ICF) core competencies served as a guide to develop the intervention and associated fidelity measures. Practicing life coaches do not require professional certification by any recognised body in the field; but all HI-DMIE life coaches completed project-mandated trainings and received coaching from a certified coach to help ensure consistent delivery of the intervention. Life coaches were full-time employees of the HI-DMIE project; therefore, a majority of their responsibilities included being available to see participants on a regular basis.

Pharmacist counselling was based on an Asheville, North Carolina project and the Diabetes Ten City Challenge (Fera, Bluml, \& Ellis, 2009). Five licensed pharmacists received training in motivational interviewing techniques, medication management and diabetes education through the project. Motivational interviewing (MI) is a strategy based on the transtheoretical model found to be effective in improving health outcomes for adults with diabetes (Smith, Heckemeyer, Kraty, \& Mason, 1997; Smith West, DiLillo, Bursac, Gore, \& Greene, 2007; Welch, Rose, \& Ernst, 2006). Five pharmacists supported participants to set attainable goals for improved lifestyle choices using an online chart to follow progress and address areas in need of improvement. They also made referrals to physicians or Certified Diabetes Educators (CDEs) when deemed necessary.

The HI-DMIE project recruited pharmacists through a partnership with a private business health consortium that compensated the retail pharmacies in which they worked by the half-hour for every study participant counselled. These pharmacists worked at different retail pharmacies throughout the island and collaborated with participants, physicians and other healthcare providers to facilitate a comprehensive approach to medication management.

The HI-DMIE intervention was unique in its pairing of life coaching and pharmacist counselling. Both approaches yielded positive results separately but no study to date offered life coaching and motivational interviewing concurrently. Participants received an average of 10 life coach and four pharmacist sessions during the intervention period, with most sessions lasting between 30 and 60 minutes. Dependent on individual need, participants determined the frequency of interactions with both the life coach and pharmacist. The intervention also provided opportunities to access nutrition counsellors, CDEs, and a 3-month fitness club membership (Mathematica Policy Research, 2009). The intervention was community-based, in that sessions occurred in public locations deemed convenient for participants. For instance, participants and life coaches often met at neighbourhood coffee shops or shopping 
malls. In some pharmacies, a separate meeting space carved out by the store helped to ensure privacy.

In addition to the pharmacist counselling and life coaching, the intervention consisted of incentives (benefits) such as reimbursements for diabetes-related medical supplies, use of a laptop computer and a wireless internet card. For participants who did not have employer-sponsored health insurance, the HI-DMIE provided coverage. The project also reimbursed co-payments for physician appointments pertaining to diabetes.

\section{Methods \\ Participants}

The overarching goal of the HI-DMIE was to forestall disability among a working population living with a chronic illness, therefore, recruitment efforts focused on those already employed. Between April and September 2008, the HI-DMIE enrolled 190 self-selected employed adults between the ages of 20-62, living on Oahu, having a diabetes diagnosis or $\mathrm{HbA} 1 \mathrm{c}$ level of $6.5 \%$ or higher, employed at least 10 hours per week for four consecutive weeks, receiving federal minimum wage or higher and not receiving Supplemental Security Income or Social Security Disability Insurance. Individuals were recruited through newspaper advertisements, human resources departments, diabetes-related public events, employer-sponsored health fairs, placards in the public transportation system, flyers at various pharmacies and word of mouth. Enrolment activities took place in various community settings.

Random assignment using an unbalanced design resulted in 128 participants assigned to the treatment and 62 assigned to the control group. ${ }^{1}$ All participants received diabetes educational materials and payments for completing surveys, other study documentation requirements and participating in focus groups. Treatment group participants had one year of access to the HI-DMIE intervention program. Our sample consisted of employed, well-educated and fairly healthy individuals (Table 1). The University of Hawai'i Institutional Review Board approved the study.

\section{Intervention Staff}

HI-DMIE evaluation staff collected information from the intervention staff to measure two treatment fidelity areas: model adherence (the extent to which program components, activities and methods were delivered as intended) and service delivery quality (the extent to which a provider approached the theoretical ideal in delivering program content). All intervention staff (nine life coaches and five pharmacists) providing program services to the treatment group participated in this aspect of the project. All life coaches and pharmacists lived on O'ahu during the study and had at least a bachelor's degree in a social science field or pharmacy, respectively. All were female with the exception of one life coach and one pharmacist.

\section{Measures}

Treatment effect. To measure psychosocial self-efficacy specific to diabetes, the Diabetes Empowerment ScaleShort Form (DES-SF) (Anderson, Fitzgerald, Gruppen, Funnell, \& Oh, 2003) was administered at baseline, six, and 12 months. The DES-SF is an eight-item survey $(\alpha=$ .84 ) that uses a 5-point Likert scale ( $1=$ strongly disagree, 5 = strongly agree) for each item. Respondents rate the extent they agree that they: (1) know what parts of taking care of their diabetes that they are dissatisfied with, (2) are able to turn their diabetes goals into a workable plan, (3) can try out different ways of overcoming barriers to their diabetes goals, (4) can find ways to feel better about having diabetes, (5) know the positive ways they cope with diabetes-related stress, (6) can ask for support for

\section{Table 1}

Baseline Characteristics

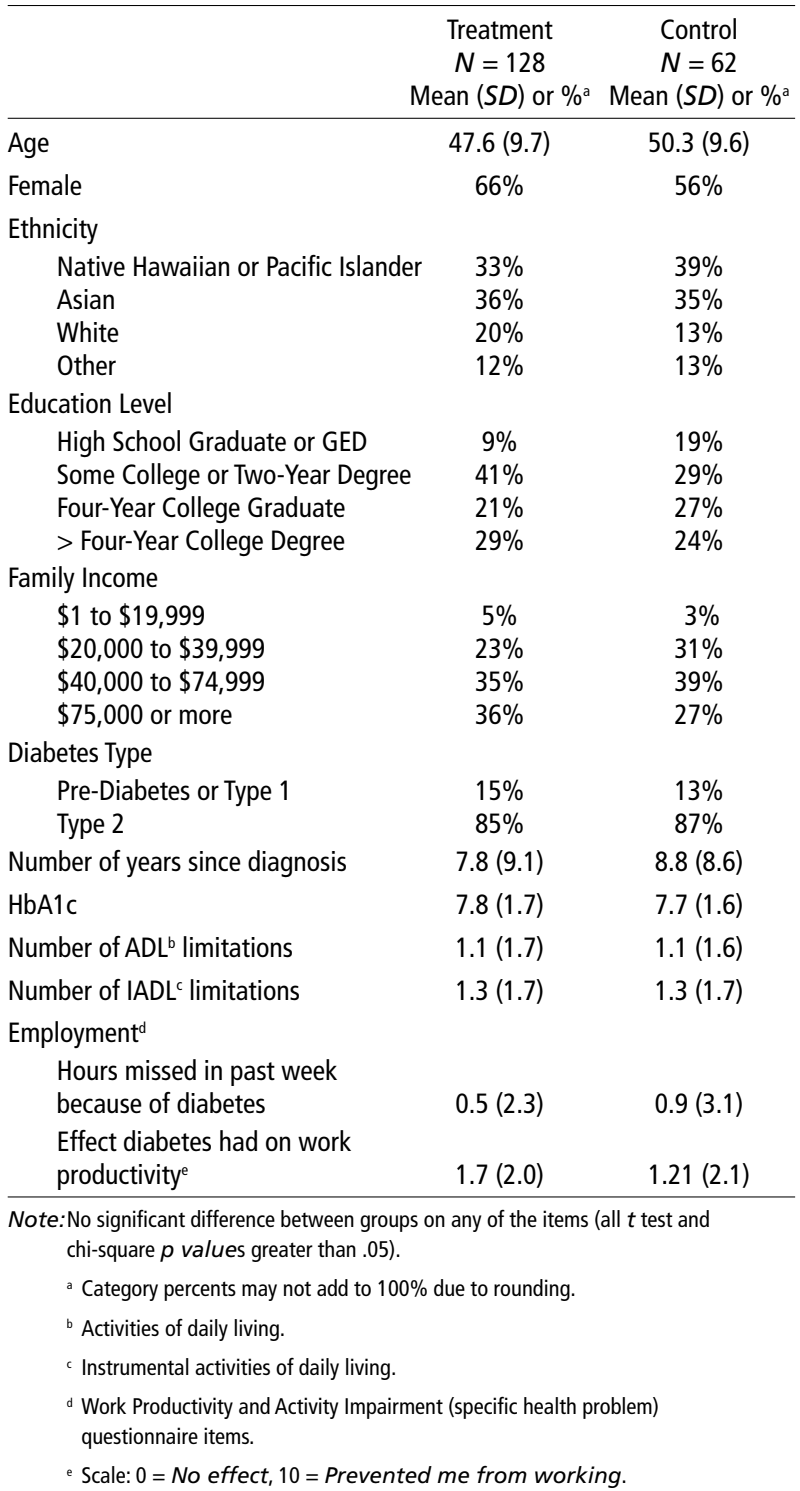


having and caring for their diabetes when they need it, (7) know what helps them stay motivated to care for their diabetes and (8) know enough about themselves as a person to make diabetes care choices that are right for them (Michigan Diabetes Research and Training Center, 2003). A DES-SF score represents the average of all survey items with higher scores indicating greater self-efficacy.

In addition, a standardised enrolment form obtained self-reported baseline information on demographics, health and employment from study participants. Participant's healthcare providers also supplied baseline height, weight and HbAlc. For treatment group participants, administrative study data collected through the project described the extent of program engagement or exposure to the intervention.

Participant perceptions. Fifty-five treatment group participants voluntarily participated in 27 focus group meetings. Eleven meetings occurred approximately six months after participants enrolled in the study, and 16 occurred 12 months after enrolment or at the end of the intervention period. The number of participants attending a meeting ranged from one ${ }^{2}$ to seven, with an average meeting size of four participants. Forty-seven participants attended the six-month meetings and 32 attended the 12-month meetings. All focus group participants consented to being audio recorded, and their responses were confidential. The facilitator and assistant also took notes during each meeting. We asked focus group participants several broad questions related to their experience with the intervention components, using standardised prompts to probe for further clarification. This article highlights the most salient findings relevant to self-management and self-efficacy. Responses described in this article pertain to the following question: 'Over the past 6/12 months, has your participation in this project affected your diabetes management in any way? If so, please explain'.

Intervention fidelity. To measure model adherence, a selfreport Likert scale survey asked life coaches and pharmacists to rate the degree to which they adhered to the program model $(1=$ no adherence, $4=$ complete adherence $)$. To measure the quality of service delivery by life coaches and pharmacists, all life coach and pharmacist meetings with participants were audio recorded throughout the intervention period. Each quarter, 10 life coach and 10 pharmacist recordings were randomly selected, transcribed and rated by three independent evaluators trained to use instruments developed specifically for the HI-DMIE study. Instrument development and reliability testing was performed during the study's 2007-2008 pilot period (feasibility study) and resulted in life coach and pharmacist instrument reliability values of .88 and .87 , respectively (Fukunaga \& Uehara, 2010). The instruments used a 5point Likert-scale $(1=$ needs work, $5=$ exemplary $)$ to rate 10 life coach behaviours and eight pharmacist behaviours (Table 2). Each quarter of the intervention period, two recordings (one life coach, one pharmacist session) were rated by all evaluators for inter-rater reliability purposes. Each evaluator also rated six of the remaining 18 selected recordings. Selected session recordings excluded the first two meetings and the final meeting due to administrative activities required in these sessions.

\section{Analysis}

Treatment effect. We used descriptive, $t$ test, and chisquare analyses to examine the baseline demographic, employment and health characteristics of participants. During outcomes analysis, to better estimate the variability and uncertainty in missing data, we performed multiple imputation that resulted in five simulated, complete versions of data used in all DES-SF analyses. Missing

\section{Table 2}

HI-DMIE Theoretical Ideals: Behaviours Rated for Life Coaching and Pharmacist Counselling

\begin{tabular}{ll}
\hline Life Coach Key Behavioural Components & Pharmacist Key Behavioural Components \\
\hline Established/Maintained Trust $^{\mathrm{a}}$ & Maintains a Positive Environment ${ }^{\mathrm{a}}$ \\
Remained Nonjudgmental $^{\mathrm{a}}$ & Builds/Maintains Rapport $^{\mathrm{a}}$ \\
Exhibited Professionalism $^{\mathrm{a}}$ & Used Active Listening Strategies $^{\mathrm{b}}$ \\
Focused on Participant Agenda $^{\mathrm{a}}$ & Remained Patient Centred $^{\mathrm{b}}$ \\
Maintained Coaching Relationship $^{\mathrm{a}}$ & Monitored Diabetes Care $^{\mathrm{b}}$ \\
Asked Questions $^{\mathrm{b}}$ & Demonstrated a Collaborative Stance $^{\mathrm{c}}$ \\
Used Active Listening Strategies $^{\mathrm{b}}$ & Discussed Goal Setting and Achievement $^{c}$ \\
Facilitated Participant Goal Development $^{\mathrm{b}}$ & Assessed Stages of Change $^{c}$ \\
Elicited Answers and Actions from Participant $^{\mathrm{b}}$ & \\
Remained Solution-Focused $^{\mathrm{b}}$ &
\end{tabular}

Note: These are the behaviours evaluated in order to assess the quality of service delivery.

a Above Basic performance

${ }^{\mathrm{b}}$ Basic performance

'Below Basic performance 
data included the baseline HbAlc on 18 (9\%) participants and the DES-SF on $39(21 \%)$ and $40(21 \%)$ participants at the six and 12-month time points, respectively. We used multiple imputation because mean, conditional mean, last observation carried forward and other types of imputation tend to reduce variance and can distort relationships among variables. These methods also treat imputed values as though they were observed, which they are not (imputations are only estimates). On the other hand, completely ignoring missing data can produce biased results. Multiple imputation addresses missing data without producing bias by imputing multiple times in order to incorporate some variation into the imputed estimates (Der \& Everitt, 2009; Little \& Rubin, 2002; Schafer, 1997). We assessed the adequacy of the number of imputations performed through the relative efficiency of using a finite number of imputations instead of infinitely many ( 0 to 1 , with higher values meaning higher efficiency). The five multiple imputation datasets resulted in a baseline $\mathrm{HbAlc}$ relative efficiency of .97 and six- and 12-month DES-SF score relative efficiencies of .98 and .99 , respectively, indicating that the advantage of additional imputations was negligible.

To examine the effect of the intervention on diabetes self-efficacy, we used the baseline DES-SF as the covariate and repeated measures analysis of covariance (ANCOVA) to identify the effect of group assignment (treatment, control) on DES-SF score as well as to estimate and compare differences in DES-SF group mean scores at the six- and 12-month time points. After calculating intraclass correlation coefficients (ICCs) to measure the effect different providers (coach and pharmacist) had on treatment group participant outcomes, we did not adjust for an effect because of the minimal influence providers had on DES-SF score variation. Six months after the intervention program started, providers accounted for less than $1 \%$ of the variation in treatment group self-efficacy scores (mean ICC $=.0011$ ). At the end of the intervention period (12-months postbaseline), the provider effect was still minimal (mean ICC $=.018$ ).

Of the five $F$ statistics calculated from the five imputed datasets, statistical significance was defined as all five corresponding $p$ values being lower than .05 . The median $F$ statistic was used to quantify the results (describe whether the treatment had an effect and whether this effect was consistent across the study period). We then compared imputed and nonimputed 12-month group differences to examine the influence multiple imputation had on results. Secondary analyses categorised treatment group members by their baseline HbAlc (diabetes control), ethnicity and exposure to the intervention and used $t$ tests to explore changes in baseline to 12-month DES-SF scores (differences between an individual's baseline and 12-month score). We used the statistical package SAS 9.2 (SAS Institute Inc.) for all analyses. Consistent with the reporting of results obtained using multiple imputation, we report standard errors rather than standard deviations when the imputed datasets were used for analysis.

Participant perceptions. We transcribed the focus group audio recordings verbatim and deleted identifying personal information during transcription. Two evaluation staff members read each transcript, independently identified frequent themes and met to reach consensus on emergent themes. One researcher coded responses to each question by using notes capturing group dynamics to identify themes that occurred most frequently. A second layer of analysis identified main categories of participant experience based on the relationships between initial emergent themes. During this stage of analysis, we kept the following distinctions in mind: (1) the number of people within a focus group who gave similar responses, (2) the number of people across different focus groups who gave similar responses and (3) when a response was given by an individual, the number of people who agreed verbally or nonverbally. We targeted our analysis on the main categories of participant experience as a best representation of what was said across focus groups.

Intervention fidelity. We used life coach and pharmacist survey responses to derive the mean adherence to the intended model. To assess quality of service delivery we calculated a mean rating for each of the 18 behaviours (10 life coach, eight pharmacist) and then categorised the delivery of each behaviour as Exemplary (4.5 or higher), Above basic (3.5 up to 4.5), Basic (2.5 up to 3.5), Below basic (1.5 up to 2.5) or Needing work (1 up to 1.5). Interrater reliability was computed using Cronbach's alpha. We used SPSS and Excel for all analyses.

\section{Results \\ Treatment Effect}

At baseline there were no variables where treatment and control group participants differed significantly. The two groups were statistically similar across age, gender, diabetes type, ethnicity, educational attainment, household income, functioning and work productivity (Table 1). The majority of participants were minorities, many of whom were Asian or Native Hawaiian or Pacific Islander. Mean DES-SF scores at baseline for the treatment and control groups were similar at $3.77(S D=0.69)$ and 3.80 $(S D=0.82)$ respectively, $t(188)=0.29, p=.77$. During the intervention period, the positive effect of the intervention on DES-SF score increased as the study progressed (Figure 1).

This effect was significant, median $F(1,187)=10.40$, $p=.002 .{ }^{3}$ However, the treatment effect was not constant across the study period, indicated by a significant group x time interaction term, median $F(1,188)=8.70$, $p=.004^{4}$. The difference in mean score between treatment and control groups was $0.13(S E=0.09, p=.16)$ and $0.35(S E=0.10, p<.001)$ at the six- and 12-month 


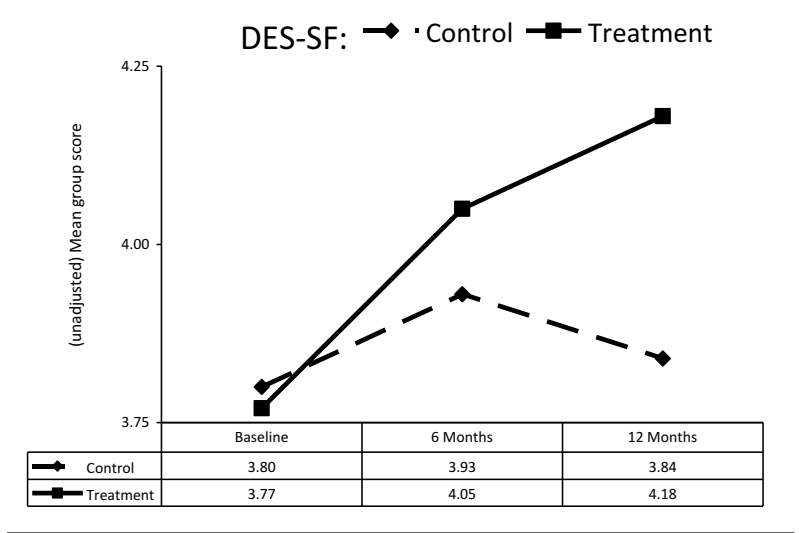

Figure 1

Mean diabetes empowerment scale scores for treatment and control group participants over the study period.

time points, respectively. Within the treatment group, significant increases in DES-SF scores were found for both genders and among the following groups: baseline HbA1c of 7 or higher, non-Asians, and those with 10 or more intervention sessions (Table 3).

\section{Participant Perceptions}

Focus groups provided personalised information to supplement and further inform the responses obtained from the self-efficacy surveys. At both six and 12 months, participants reported making psychological and behavioural self-management changes as well as health improvements. The questions asked in the focus groups identified the following themes pertaining to perceived treatment effects: psychosocial changes, diabetes selfmanagement changes and health improvements. At six months, $54 \%$ of focus group responses reflected a change in psychosocial functioning and this theme was discussed most frequently. Further defining psychological changes at six months, participants identified the following: increased motivation, greater awareness of diabetes, enhanced diabetes self-efficacy and improved self-awareness. At 12 months, participants most frequently reported making behavioural self-management changes, followed by psychosocial changes and health improvements. When asked to further describe their psychosocial improvements, enhanced diabetes self-efficacy was discussed most frequently followed by greater awareness of diabetes, improved self-awareness and increased motivation (to make changes). The following quotes provide an example of the psychosocial theme that emerged from focus group participants:

What I have learned from this project is that my diabetes was manageable, that I could control it. It was all up to me.

I just feel like I'm more in control of my body.

Most importantly to me though, was that I learned that I had to be in control of everything and it was my responsibility to make sure that I did all of the things that I was supposed to do.

Table 3

Change in Treatment Group DES-SF Score by Gender, HbA1c, Ethnicity and Exposure Level

\begin{tabular}{|c|c|c|c|c|c|c|c|c|}
\hline & \multicolumn{4}{|c|}{ Mean DES-SF score } & \multirow[b]{2}{*}{$S E$} & \multirow[b]{2}{*}{$d f$} & \multirow[b]{2}{*}{$t$ value } & \multirow[b]{2}{*}{$p$ value } \\
\hline & $N$ & Baseline & 12-months & Difference & & & & \\
\hline \multicolumn{9}{|l|}{ Gendera } \\
\hline Female & 84 & 3.81 & 4.22 & 0.41 & 0.08 & 192.2 & 5.05 & $<.0001$ \\
\hline Male & 44 & 3.70 & 4.11 & 0.42 & 0.13 & 112.1 & 3.10 & .003 \\
\hline \multicolumn{9}{|c|}{ Baseline $H b A 1 c^{b, c}$} \\
\hline$<7$ & 39 & 4.11 & 4.29 & 0.19 & 0.13 & 314.1 & 1.48 & .14 \\
\hline 7 to 9 & 61 & 3.61 & 4.14 & 0.53 & 0.10 & 331.3 & 5.45 & $<.0001$ \\
\hline$>9$ & 28 & 3.65 & 4.13 & 0.48 & 0.16 & 40.4 & 3.06 & .004 \\
\hline \multicolumn{9}{|l|}{ Ethnicity ${ }^{\mathrm{b}}$} \\
\hline Asian & 47 & 3.86 & 4.19 & 0.33 & 0.13 & 29.4 & 2.45 & .02 \\
\hline $\mathrm{NHPl}^{\mathrm{d}}$ & 42 & 3.73 & 4.06 & 0.33 & 0.12 & 928.5 & 2.82 & .005 \\
\hline Other ${ }^{\mathrm{e}}$ & 39 & 3.71 & 4.31 & 0.60 & 0.12 & 101.6 & 4.85 & $<.0001$ \\
\hline \multicolumn{9}{|c|}{ Number of sessions ${ }^{b}$} \\
\hline 9 or fewer & 29 & 3.72 & 3.95 & 0.24 & 0.18 & 42.3 & 1.35 & .19 \\
\hline 10 to 19 & 69 & 3.87 & 4.26 & 0.39 & 0.07 & 791.6 & 5.32 & $<.0001$ \\
\hline 20 or more & 30 & 3.59 & 4.22 & 0.63 & 0.16 & 29 & 3.94 & $<.001$ \\
\hline
\end{tabular}

Note: Paired $t$-test using difference between 12 month and baseline score.

a $p$ values less than .025 are statistically significant (Bonferroni correction for two comparisons: .05/2).

${ }^{b} p$ values less than .017 are statistically significant (Bonferroni correction for three comparisons: .05/3).

c Sample sizes shown are the median of the five imputations.

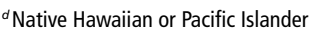

e There were not enough 'White' treatment group participants to analyse separately; 'White' participants are included in the 'Other'category. 
I mean I really feel empowered ... I think this program was very positive. It really helped me learn about my goals and ... what are my options and just to think about like alternatives. ...

Intervention fidelity. Eight (89\%) life coaches and two (40\%) pharmacists responded to the intervention staff survey intended to measure model adherence (the extent to which program methods were delivered as intended). Both life coaches and pharmacists reported performing the key functions in delivering the program as intended with high to moderate adherence. With a value of one (1) indicating no adherence to the model and a value of four (4) indicating complete adherence, the life coach mean adherence was $3.25(S D=0.46)$; the pharmacist mean adherence was $3.00(S D=0)$.

The quality of service delivery was measured by rating the performance of the theoretical ideals listed in Table 2. Life coaches performed all 10 behaviours at the basic $(n=5)$ or above basic $(n=5)$ level; Pharmacists performed five of eight $(62 \%)$ behaviours at the basic $(n$ $=3)$ or above basic $(n=2)$ level. Life coach and pharmacist scale instrument interrater reliability computed using Cronbach's alpha were .80 and .79 , respectively. Additional and more detailed intervention fidelity results can be found in Fukunaga and Uehara (2010).

\section{Discussion}

Our findings suggest that well-educated and wellemployed adults from diverse backgrounds significantly improved their diabetes self-efficacy when provided life coaching and pharmacist counselling. Administrative data and focus group findings indicated that treatment group members engaged in managing their chronic illness and participated in services specifically geared to their lifestyles. Additionally, higher levels of intervention program participation (more life coach and pharmacist sessions) resulted in higher self-efficacy gains within the treatment group.

Adults are intrinsically motivated to learn; however, that motivation stems from individual views about how given material relates to their adult roles. Learning imposed on adults often results in resentment and is minimally effective (Knowles, 1984). Adult learner characteristics such as age, literacy level, culture, autonomy and personal choice are factors to consider when tailoring interventions (Walker, 1999). Motivational interviewing techniques used by the pharmacists assessed conviction and confidence two components of motivation that can be used to gauge an individual's readiness to engage in health behaviours (Koenigsberg, Bartlett, \& Cramer, 2004). Supporting a diverse employed adult population living with a chronic illness resulted in the development of a flexible, individualised community-based intervention.

Within this diverse sample, personalised interactions provided a foundation to elicit empowerment beliefs toward behaviour change. Using multiple techniques to address self-efficacy, coaches and pharmacists often engaged in a 'talk story' format, an informal conversation style as a way to build trust and relationship (Taosaka, 2002), before discussing health or work issues. This approach required time to work and may be one of the reasons why the effect of the intervention on diabetes self-efficacy was not yet evident at six months. Treatment group analysis showed a positive response to the intervention for both genders, those with higher baseline HbAlc levels and for those in certain ethnicities. For example, treatment participants who were not in control of their diabetes (HbAlc of 7 or higher) and Native Hawaiian/Pacific Islanders showed significant self-efficacy gains over the course of the study. These findings provide support for the intervention with specific, targeted populations where access to effective programs is critical. In addition, the intervention was successful for both males and females, important results to note for future program implementation.

The personal model of life coaching and pharmacist counselling revealed a promising strategy to address selfefficacy among an employed minority populace. However, the sample represented a fraction of the Pacific population living with diabetes and it did not include disadvantaged individuals. For example, the study sample was limited to residents on the island of O'ahu and therefore not representative of individuals with diabetes in the vast Pacific. In addition, the study required participants to be employed at baseline, which resulted in no study representation for unemployed individuals with diabetes. Finally, the study sample comprised individuals who volunteered to participate in the random assignment trial. This resulted in a study sample that was more motivated than would have been had random selection taken place (Nishita, Uehara, \& Tom, 2011).

Focus group findings support the DES-SF results but do not mirror the trend of a constant increase in self-efficacy across the study period. During the intervention period, participants identified psychosocial changes including self-efficacy as an important aspect of their selfmanagement behaviours at month six. As the study progressed the positive effect of the intervention on DESSF score increased and the frequency of discussing psychosocial changes decreased. This signifies a logical progression of behaviour change. During the intervention period, psychological change was the highest ranked theme regarding treatment effects, followed by behavioural and physical improvements, respectively. At the end of the intervention period, the first and second rankings reversed. This may indicate that before any behavioural and physical changes can take place, a conscious shift in one's thinking is needed. Focus group findings also highlighted consistency in responses among the various participants. In other words, although there were sometimes different participants in the six- and 12-month focus groups, their responses to the same question were 
similar. Finally, because focus group participants were not representative of the entire treatment group, broad generalisations of the findings should be avoided.

Because the HI-DMIE consisted of testing the effects of a newly developed intervention, measuring treatment fidelity was an essential research component. Both internal and external validity of the study depended on the rigour of our measures. Assessing model adherence and quality of service delivery documented the utility of the treatment and determined if the results were a function of the treatment or some other factors.

We found that the program model was executed quite well overall, with life coaches and pharmacists providing services that adhered to the intended model. Based on the life coaches' self-reported adherence to the model and the external service delivery quality performance ratings, we determined that the life coaches implemented the treatment condition with greater fidelity than the pharmacists. We speculated that this finding might be due to the life coaches' full-time employment with the research study in which they exclusively focused on delivering a consistent coaching model to all treatment participants and their employment depended on the study. The pharmacists, on the other hand, were employed by private, retail stores where the study was just a small portion of their workload and their employment did not depend on the study. During the study period, pharmacists counselled both research participants and general public clients, perhaps resulting in an unintended dilution or modification of the service delivery model to accommodate the numerous clients needing one-to-one attention. Finally, note that only two of the five pharmacists responded to the intervention staff (adherence) survey, therefore this particular aspect of the fidelity results may not be representative of what actually occurred during the study.

The study did not individually test treatment components; therefore, we cannot identify the extent to which pharmacist support or life coaching made the difference in self-efficacy. In addition, baseline DES-SF scores were fairly high, indicating a sample that, at study onset, was already somewhat confident in their ability to manage the disease. Although most participants appeared to be scoring at the top of the scale with not much room for improvement at baseline, treatment group members demonstrated a significant gain as compared with the control group - we view these self-efficacy findings as remarkable. However, we acknowledge that the study was unblinded and treatment group participants received more attention from the study than control group participants, which might have influenced results. The programmatic costs associated with these self-efficacy gains are unclear, because the HI-DMIE project did not include a cost-effectiveness or cost-benefit component. Moreover, because the HI-
DMIE did not track participants much beyond the intervention period, we were unable to examine long-term reductions in healthcare services or reduced reliance on federal assistance programs.

While self-efficacy has been shown to predict performance, (Stajkoric \& Luthans, 1998) with only six months separating most data-collection points, detection of changes over time was a consistent challenge. The 12-month duration of the intervention may not have been enough to impact physical changes. Furthermore, life experiences and personal values were not captured in this study and perhaps play a large role in how one enacts self-management behaviours that self-efficacy often precedes.

The strength of the study was effective delivery of a multicomponent, individually tailored treatment to an ethnically diverse sample of diabetics. Results of this study provide preliminary evidence on the effectiveness of community-based, individualised services in raising self-reported diabetes self-efficacy within a well, employed, ethnically diverse population of adults with diabetes. We can be confident, to some extent, that the results were due to the intervention because of the rigorous study design and because fidelity findings indicate the program was implemented as planned. Both life coaches and pharmacists delivered the intervention with acceptable levels of integrity which, in turn, reflected measurable changes in how participants thought about their disease. We suggest that additional and future research include: (a) a longer study (tracking) period, (b) individuals at higher risk for diabetes complications and disability, (c) individuals who may be economically or socially disadvantaged and/or have limited access to diabetes management resources, (d) an examination of the separate intervention components and (e) the costeffectiveness or benefit of the intervention program itself. To reduce the influence of the Hawthorne effect on results, we recommend the inclusion on non-selfreported outcome measures, a control group program that provides a similar amount of attention to participants and a blinded study setting.

\section{Implications for Practice}

These findings support several implications for diabetes management programs for working adults. Our data confirms that employed adults with diabetes respond to community-based individually tailored one-to-one services facilitating individual goal setting. During the intervention period, the positive effect of the intervention on treatment participants' diabetes self-efficacy improved while control group participants' self-efficacy remained fairly stable. Secondary analysis suggests that even moderate interaction can lead to positive changes. Particularly for diverse, minority populations, efforts to build self-confidence through concerted relationship- 
building appear to pay off in terms of the investment of time from both the service provider and client.

Diabetes management programs for working adults could utilise life coaches to provide a nonthreatening, self-paced format to promote attitudinal changes. In addition, persons with diabetes should have regular reinforcement to sustain a high level of self-efficacy in managing their disease. The findings of Norris and colleagues (2001) found that one shot or short-term education proved less effective in diabetes management. Our findings demonstrate the value of sustained personalised coaching activities.

We realise that in the Pacific region beyond Hawai $i$, persons with diabetes may not have the same advantages as HI-DMIE study participants, such as access to coaches and pharmacists, employer-sponsored health insurance, healthcare options or consistent employment. In these environments, strategies such as the personalised approach used by the life coaches could be adapted to fit within a clan or village setting. Appropriate family members or highly respected village leaders could take on the role of 'coach' understanding that the health of individual members contributes to the wellness of the community. Through community/village meetings the larger group could also serve as a support system by: (1) providing an information-sharing vehicle about topics such as healthy eating and (2) allowing a forum for individuals to share disease management strategies.

\section{Endnotes}

1 In an effort to attract potential participants, the project director requested that the allocation of random assignment favour those assigned to the treatment group. For every nine participants assigned, six were randomly assigned to treatment and three were randomly assigned to control.

2 Three focus groups had only one participant in attendance as a result of no shows or cancellations by the others. In these cases individual interviews were conducted using the same protocols and procedures (questions and prompts) used in the focus groups.

3 Median ANCOVA statistics shown; all five ANCOVA $p$ values less than .05 (range $=.0005$ to .02 ).

4 Median ANCOVA statistics shown; all five ANCOVA $p$ values less than .05 (range $=.0003$ to .01 ).

\section{Funding}

This work was supported by the Centers for Medicare and Medicaid Services [grant number CFDA No. 93.769].

\section{References}

Anderson, R.M., Fitzgerald, J.T., Gruppen, L.D., Funnell, M.M., \& Oh, M.S. (2003). The Diabetes Empowerment ScaleShort Form (DES-SF). Diabetes Care, 26, 1641-1643.

Bandura, A. (1994). Self-efficacy. In V.S. Ramachaudran (Ed.), Encyclopedia of human behavior (Vol. 4, pp. 71-81). New York: Academic Press. (Reprinted in H. Friedman [Ed.],
Encyclopedia of mental health. San Diego: Academic Press, 1998). Retrieved from http://www.des.emory.edu/mfp/ BanEncy.html

Der, G., \& Everitt, B.S. (2009). A handbook of statistical analysis using SAS. Boca Raton, FL: Chapman \& Hall.

Fera, T., Bluml, B.M., \& Ellis, W.M. (2009). Diabetes Ten Cities challenge: Final economic and clinical results. Journal of the American Pharmacists Association, 49(3), e52-e60.

Fukunaga, L., \& Uehara, D. (2010, June). Innovations in Treatment Fidelity: Measuring Quality of Implementation for a Life Coaching and Pharmacist Counseling Intervention for Employed Diabetics. Poster presented at the 2010 AcademyHealth Annual Research Meeting, Boston, MA. Retrieved from http://manoa.hawaii.edu/livehealthy/ Presentations/ADA2010/DMIEPoster.pdf

Johnston-Brooks, C.H., Lewis, M.A., \& Garg, S. (2002). Selfefficacy impacts self-care and HbAlc in young adults with Type I diabetes. Psychosomatic Medicine, 64(1), 43-51.

Knowles, M.S. (1984). Introduction: The art and science of helping adults learn. In Andragogy in action: Applying modern principles of adult learning. San Francisco: Jossey-Bass.

Koenigsberg, M.R., Bartlett, D., \& Cramer, J.S. (2004). Facilitating treatment adherence with lifestyle changes in diabetes. American Family Physician, 69(2), 309-316.

Krichbaum, K., \& Buethe, M. (2003). Exploring the connection between self-efficacy and effective diabetes selfmanagement. Diabetes Educator, 29, 653-662.

Little, R.J.A, \& Rubin, D.B. (2002). Statistical analysis with missing data (2nd ed.). Hoboken, NJ: John Wiley \& Sons, Inc.

Mathematica Policy Research. (2009). Interim report on the Demonstration to Maintain Independence and Employment. Retrieved from http://www.mathematica-mpr.com/publications/PDFs/disability/dmie-interimrpt.pdf

Michigan Diabetes Research and Training Center. (2003). Diabetes Empowerment Scale-Short Form (DES-SF). Retrieved from: http://www.med.umich.edu/mdrtc/ profs/survey.html

Nishita, C.M., Uehara, D.L., \& Tom, T. (2011). Diabetes selfmanagement support needs and concerns for the future among employed adults on Oahu. Hawaii Journal of Public Health, 3(1), 30-35.

Norris, S.L., Engelgau, M.M., \& Narayan, K.M. (2001). Effectiveness of self-management training in Type 2 diabetes: a systematic review of randomized controlled trials. Diabetes Care, 24, 561-587.

Sarkar, U., Fisher, L., \& Schillinger, D. (2006). Is self-efficacy associated with diabetes self-management across race/ ethnicity and health literacy? Diabetes Care, 29, 823-829.

Satia, J.A., \& Galanko, J.A. (2007). Intrinsic and extrinsic motivations for healthful dietary change in African Americans. American Journal of Health Behavior, 31(6), 643-655.

Schafer, J.L. (1997). Analysis of incomplete multivariate data. London, UK: Chapman \& Hall.

Smith, D.E., Heckemeyer, C.M., Kraty, P.P., \& Mason, D.A. (1997). Motivational interviewing to improve adherence to a behavioral weight-control program for older obese women with NIDDM. Diabetes Care, 20, 52-54. 
Smith West, D., DiLillo, V., Bursac, Z., Gore, S.A., \& Greene, P.G. (2007). Motivational interviewing improves weight loss in women with Type 2 diabetes. Diabetes Care, 30(5), 1081-1087.

Spigner-Littles, D., \& Anderson, C. E. (1999). Constructivism: A paradigm for older learners. Educational Gerontology, 25, 203-209.

Sprague, M., Shultz, J., \& Branen, L. (2006). Understanding patient experiences with goal setting for diabetes self-management after diabetes education. Family \& Community Health, 29(4), 245-255.

Stajkoric, A.D., \& Luthans, F. (1998). Self-efficacy and work related performance: A meta-analysis. Psychological Bulletin, 124, 240-261.
Taosaka, S. (2002). Let's talk story: Professional development in the Pacific. Retrieved from http://www.prel.org/products/ re_/talkstory.htm

Walker, E. (1999). Characteristics of the adult learner. The Diabetes Educator, 25, 16-24.

Welch, G., Rose, G., \& Ernst, D. (2006). Motivational interviewing and diabetes: What is it, how is it used, and does it work? Diabetes Spectrum, 19(1), 5-11.

Whittemore, R., Melkus, G.D., \& Grey, M. (2002). Metabolic control, self-management and psychosocial adjustment in women with Type 2 diabetes. Journal of Clinical Nursing, 14, 195-203. 